\title{
Estimation of the Rayleigh Distribution under Unified Hybrid Censoring
}

\author{
Young Eun Jeon \\ Dept. of Statistics, \\ Yeungnam University
}

\author{
Suk-Bok Kang \\ Dept. of Statistics, \\ Yeungnam University
}

\begin{abstract}
We derive some estimators of the scale parameter of the Rayleigh distribution under the unified hybrid censoring scheme. We also derive some estimators of the reliability function and the entropy of the Rayleigh distribution. First, we obtain the maximum likelihood estimator of the scale parameter. Second, we obtain the Bayes estimator using the mean of the posterior distribution. Lastly, we obtain the Bayes estimator using the mode of the posterior distribution. We also derive the interval estimation (confidence interval, credible interval, and HPD credible interval) for the scale parameter under the unified hybrid censoring scheme. We compare the proposed estimators in the sense of the mean squared error through Monte Carlo simulation. Coverage probability and average lengths of $95 \%$ and $90 \%$ intervals are obtained.
\end{abstract}

Keywords: Bayes estimator, credible interval, confidence interval, entropy, maximum likelihood estimator, Rayleigh distribution, reliability function, unified hybrid censoring.

\section{Introduction}

Consider a Rayleigh distribution with the probability density function (pdf) :

$$
f(x ; \sigma)=\frac{x}{\sigma^{2}} \exp \left(-\frac{x^{2}}{2 \sigma^{2}}\right),
$$

and the cumulative distribution function (cdf) :

$$
F(x ; \sigma)=1-\exp \left(-\frac{x^{2}}{2 \sigma^{2}}\right), \quad x>0, \sigma>0 .
$$

Also, the reliability function of the Rayleigh distribution is

$$
R(t \mid \sigma)=1-F(t ; \sigma)=P[X>t]=\exp \left(-\frac{t^{2}}{2 \sigma^{2}}\right), \quad t>0
$$


For the pdf (1), the entropy simplifies to :

$$
H(f)=1+\ln \left(\frac{\sigma}{\sqrt{2}}\right)+\frac{\gamma}{2}
$$

where $\gamma$ is the Euler-Mascheroni constant.

The Rayleigh distribution is widely used in communication engineering and life testing of electro vacuum devices. So, the Rayleigh distribution has been discussed by many researchers as a lifetime distribution. Balakrishnan (1989) proposed an approximate maximum likelihood estimator (AMLE) of the scale parameter of the Rayleigh distribution with the censoring scheme. Fernandez (2000) studied the Bayesian inference from the Type-II doubly censored Rayleigh samples. Wu, Chen, and Chen (2006) studied the Bayesian inference for the Rayleigh distribution under the progressive censored samples. Han and Kang (2006) discussed the estimation for the two-parameters Rayleigh distribution based on the multiple Type-II censored samples. Kim and Han (2009) studied the estimation of the scale parameter of the Rayleigh distribution under the general progressive censoring scheme. Raqab and Madi (2011) discussed the inference for the generalized Rayleigh distribution based on the progressive censored samples. Kang, Cho, Han, and Kim (2012) studied the estimation of the entropy for a double exponential distribution based on the multiply Type-II censored samples. Abdel-Hamid and AL-Hussaini (2014) discussed the Bayesian prediction for Type-II progressive censored samples from the Rayleigh distribution under the progressive-stress model. Cho, Sun, and Lee (2014) proposed the estimations of the entropy for the Rayleigh distribution based on the doubly-generalized Type-II hybrid censored samples. Seo and Kang (2015) discussed the Bayesian estimation of the entropy of the half logistic distribution based on the Type-II censored samples. Kundu and Raqab (2015) obtained the estimation of $R=P[Y<X]$ for the three-parameters generalized Rayleigh distribution. Dey, Dey, and Kundu (2016) studied the two-parameters Rayleigh distribution under the progressive Type-II censoring scheme. Seo, Jeon, and Kang (2016) proposed the exact interval inference for the two-parameters Rayleigh distribution based on the upper record values. Chaturvedi, Kang, and Pathak (2016) studied the estimation and testing procedures for the reliability functions of the generalized half logistic distribution. Seo and Kang (2017) proposed more efficient inference using the hybrid censored samples from a continuous probability distribution with the scale parameter.

In the Type-I censoring scheme, the test is terminated at fixed $T \in(0, \infty)$ and uses the observed samples until that $T$. In the Type-II censoring scheme, the test is terminated when samples are observed until the predetermined observation number $(r)$. These were combined by Childs, Chandrasekar, Balakrishnan, and Kundu (2003) and are called the hybrid censoring scheme that also has two types. Chandrasekar, Childs, and Balakrishnan (2004) combined these two types as the generalized Type-I hybrid censoring scheme and generalized Type-II hybrid censoring scheme. A mixture of generalized Type-I and Type-II hybrid censoring scheme is known as the unified hybrid censoring scheme combined by Balakrishnan, Rasouli, and Sanjari Farsipour (2008). They proposed exact likelihood inference based on the unified hybrid censoring scheme from the exponential distribution. Panahi and Sayyareh (2016) studied the estimation and prediction for the Burr Type XII distribution based on the unified hybrid censoring scheme. Ateya (2017) discussed the estimations under the inverse Weibull distribution based on the unified hybrid censoring scheme. Kaushik (2019) proposed a progressive interval Type-I censored life test plan for the Rayleigh distribution.

In this paper, we derive some estimators of the scale parameter of the Rayleigh distribution under the unified hybrid censoring scheme. The reliability function and the entropy are also estimated. In section 2, unified hybrid censoring is briefly explained. In section 3 , the scale parameter is estimated by the three methods and the interval estimation for the scale parameter is also derived. In section 4 and 5, the corresponding reliability function and entropy are estimated, respectively. Finally, the proposed methods are examined through the Monte Carlo simulation. 


\section{Unified hybrid censoring}

The lifetime of experimental objects is observed gradually and can be expressed by $X_{1: n}, X_{2: n}$, $\ldots, X_{n: n}$. Let $k$ be the object number that must be observed, $T_{1}$ be the predetermined experiment end time, and $T_{2}$ be the extended experiment end time. Let $D_{i}(i=1,2)$ be the number of observed objects until $T_{i}(i=1,2)$ and $r$ be the predetermined observation number. That is, it is assumed that $k<r<n$. In accordance with the end point and observed number, unified hybrid censoring scheme is divided into six cases.

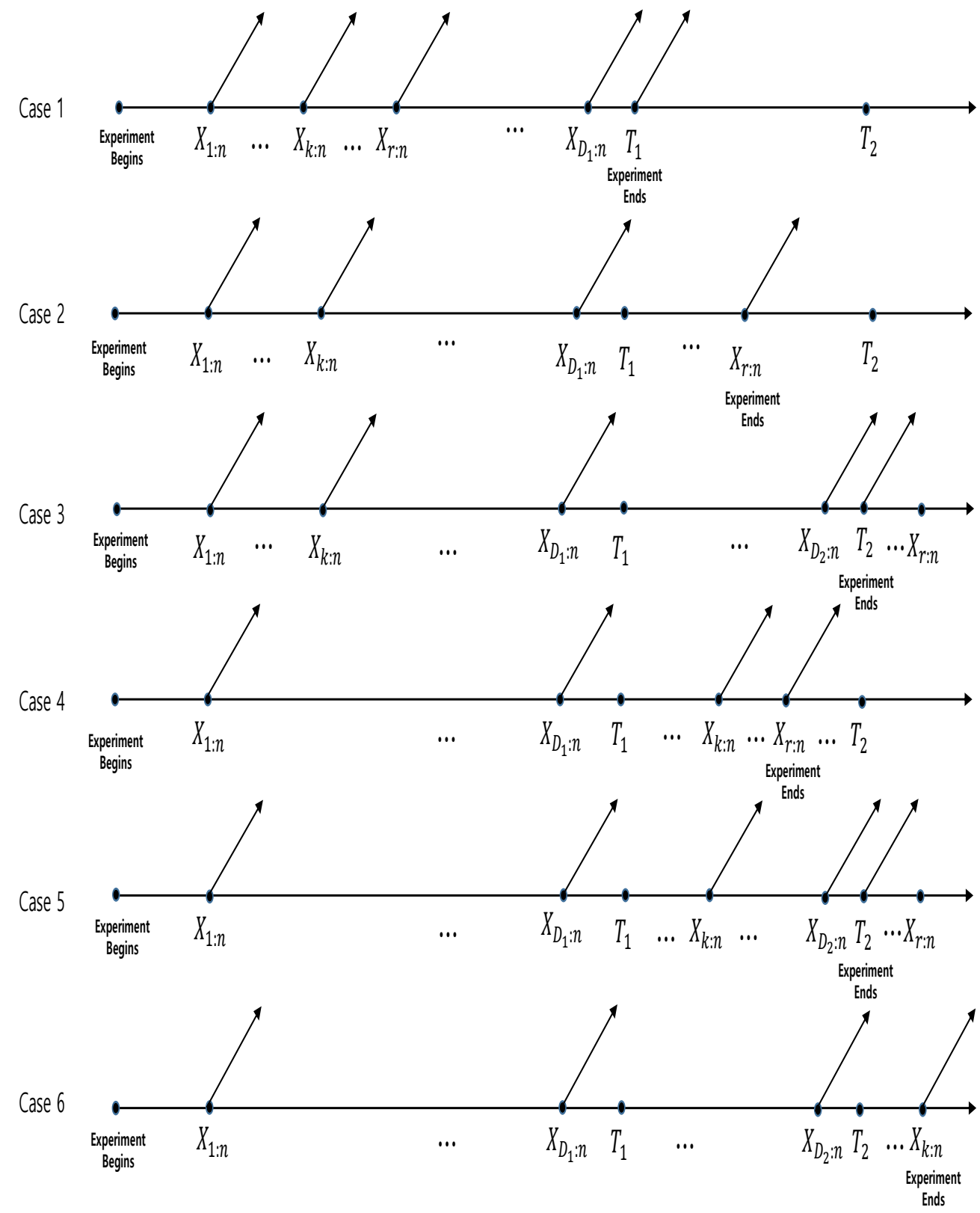

Figure 1: Unified hybrid censoring.

Case 1: $0<X_{k: n}<X_{r: n}<T_{1}<T_{2}$, the experiment ends at the point of $T_{1}$.

Case 2: $0<X_{k: n}<T_{1}<X_{r: n}<T_{2}$, the experiment ends at the point of $X_{r: n}$.

Case 3: $0<X_{k: n}<T_{1}<T_{2}<X_{r: n}$, the experiment ends at the point of $T_{2}$.

Case 4: $0<T_{1}<X_{k: n}<X_{r: n}<T_{2}$, the experiment ends at the point of $X_{r: n}$.

Case 5: $0<T_{1}<X_{k: n}<T_{2}<X_{r: n}$, the experiment ends at the point of $T_{2}$.

Case 6: $0<T_{1}<T_{2}<X_{k: n}<X_{r: n}$, the experiment ends at the point of $X_{k: n}$. 
When the $k$-th observation occurs before $T_{1}$, the experiment is terminated at $\min \left(\max \left\{X_{r: n}, T_{1}\right\}, T_{2}\right)$ and when the $k$-th observation occurs between $T_{1}$ and $T_{2}$, the experiment terminates at $\min \left(X_{r: n}, T_{2}\right)$. If it occurs after $T_{2}$, the experiment is terminated at $X_{k: n}$. The likelihood functions based on the unified hybrid censored samples are

$$
\begin{aligned}
& L_{1}(\sigma)=\frac{n !}{\left(n-D_{1}\right) !} \prod_{i=1}^{D_{1}} f\left(x_{i: n}\right)\left[1-F\left(T_{1}\right)\right]^{n-D_{1}}, D_{1}=D_{2}=D=r, \cdots, n, \\
& L_{2}(\sigma)=\frac{n !}{(n-r) !} \prod_{i=1}^{r} f\left(x_{i: n}\right)\left[1-F\left(x_{r: n}\right)\right]^{n-r}, D_{1}=k, \cdots, r-1 ; D_{2}=r, \\
& L_{3}(\sigma)=\frac{n !}{\left(n-D_{2}\right) !} \prod_{i=1}^{D_{2}} f\left(x_{i: n}\right)\left[1-F\left(T_{2}\right)\right]^{n-D_{2}}, D_{1}=k, \cdots, r-1 ; D_{2}=k, \cdots, r-1 ; \\
& D_{1} \leq D_{2}, \\
& L_{4}(\sigma)=\frac{n !}{(n-r) !} \prod_{i=1}^{r} f\left(x_{i: n}\right)\left[1-F\left(x_{r: n}\right)\right]^{n-r}, D_{1}=0, \cdots, k-1 ; D_{2}=r, \\
& L_{5}(\sigma)=\frac{n !}{\left(n-D_{2}\right) !} \prod_{i=1}^{D_{2}} f\left(x_{i: n}\right)\left[1-F\left(T_{2}\right)\right]^{n-D_{2}}, D_{1}=0, \cdots, k-1 ; D_{2}=k, \cdots, r-1, \\
& L_{6}(\sigma)=\frac{n !}{(n-k) !} \prod_{i=1}^{k} f\left(x_{i: n}\right)\left[1-F\left(x_{k: n}\right)\right]^{n-k}, D_{2}=0, \cdots, k-1 .
\end{aligned}
$$

For the above likelihood functions, we can rewrite the unified likelihood function as follows.

$$
L(\sigma ; \mathbf{x}) \propto \prod_{i=1}^{m} f\left(x_{i: n}\right)[1-F(C)]^{n-m},
$$

where $C$ is the experiment end time and $m$ is the observed number until the experiment end time $C$.

\section{Parametric estimation}

\subsection{Maximum likelihood estimation}

Using the likelihood function (6), we can obtain the log-likelihood function as follows.

$$
\ln L(\sigma ; \mathbf{x}) \propto \sum_{i=1}^{m} \ln f\left(x_{i: n}\right)+(n-m) \ln [1-F(C)] .
$$

Then, we can easily obtain the maximum likelihood estimator (MLE) using equation (7).

$$
\hat{\sigma}_{M}=\sqrt{\frac{\sum_{i=1}^{m} x_{i: n}^{2}+(n-m) C^{2}}{2 m}} .
$$

To construct the 100(1- $\alpha) \%$ confidence interval (CI) for $\sigma$, we can use the asymptotic normality of the MLE with $\operatorname{Var}\left(\hat{\sigma}_{M}\right)$ estimated from the inverse of the Fisher information. However, it is complicated to calculate the exact expected values in the Fisher information. So we derive the asymptotic variance by using the observed Fisher information

$$
I(\sigma) \approx-\frac{\partial^{2} \ln L}{\partial \sigma^{2}}=\frac{D}{\sigma^{2}},
$$


where

$$
D=-2 m+\frac{3\left(\sum_{i=1}^{m} x_{i: n}^{2}+(n-m) C^{2}\right)}{\sigma^{2}} .
$$

Under some mild regularity conditions, $\hat{\sigma}_{M}$ is approximately normal with mean $\sigma$ and variance $\operatorname{Var}\left(\hat{\sigma}_{M}\right)=1 / I\left(\hat{\sigma}_{M}\right)$. Hence, we can obtain the approximate CI based on the MLE for the scale parameter $\sigma$ as

$$
\hat{\sigma}_{M} \pm z_{\alpha / 2} \sqrt{\hat{\operatorname{Var}}\left(\hat{\sigma}_{M}\right)},
$$

where $z_{\alpha / 2}$ denotes the percentile of the standard normal distribution with right-tail probability $\alpha / 2$.

\subsection{Bayesian estimation}

For the Bayesian estimation, we consider the natural conjugate family of the prior distribution of $\sigma$ used by Fernandez (2000), given by

$$
\pi(\sigma) \propto\left(\frac{1}{\sigma}\right)^{2 \delta+1} \exp \left(-\frac{\beta}{2 \sigma^{2}}\right), \quad \sigma>0
$$

where $\delta>0$ and $\beta>0$. For $\beta=0$ in (11), $\pi(\sigma)$ reduces to a general class of improper priors. This prior distribution is known as the square-root inverted gamma distribution. We can derive the posterior distribution by combining (6) and (11) as follows.

$$
\pi(\sigma \mid \mathbf{x})=\frac{\left(\sum_{i=1}^{m} x_{i: n}^{2}+(n-m) C^{2}+\beta\right)^{m+\delta}}{2^{m+\delta-1} \Gamma(m+\delta)} \sigma^{-2 m-2 \delta-1} e^{-\frac{1}{2 \sigma^{2}}\left[\sum_{i=1}^{m} x_{i: n}^{2}+(n-m) C^{2}+\beta\right]} .
$$

Then, the Bayes estimator under the squared error loss function of $\sigma$ is obtained as

$$
\hat{\sigma}_{B}=E(\sigma \mid \mathbf{x})=\sqrt{\frac{\sum_{i=1}^{m} x_{i: n}^{2}+(n-m) C^{2}+\beta}{2}} \frac{\Gamma\left(m+\delta-\frac{1}{2}\right)}{\Gamma(m+\delta)} .
$$

We also consider the highest posterior density (HPD) estimator that is another method in popular use from the Bayesian perspective. This HPD estimator is obtained based on the maximum likelihood principle. Hence, the HPD estimator is the mode of the posterior distribution. Using the posterior distribution (12), we have

$$
\begin{aligned}
\frac{\partial \ln \pi(\sigma \mid \mathbf{x})}{\partial \sigma} & =-\frac{2 \delta+2 m+1}{\sigma}+\frac{\sum_{i=1}^{m} x_{i: n}^{2}+(n-m) C^{2}+\beta}{\sigma^{3}} \\
& =0 .
\end{aligned}
$$

We can obtain the HPD estimator by solving equation (14) as

$$
\hat{\sigma}_{H}=\sqrt{\frac{\sum_{i=1}^{m} x_{i: n}^{2}+(n-m) C^{2}+\beta}{2 m+2 \delta+1}} .
$$

In addition, we can also estimate $100(1-\alpha) \%$ credible interval (CrI) as follows.

$$
\int_{a}^{b} \pi(\sigma \mid \mathbf{x}) d \sigma=1-\alpha
$$


where $a$ denotes the percentile of the posterior distribution with left-tail probability $\alpha / 2$ and $b$ denotes the percentile of the posterior distribution with right-tail probability $\alpha / 2$. This interval has equal tail-probability. If the posterior distribution is unimodal, a common choice is the shortest connected CrI, namely the HPD interval. The HPD CrI is as follows.

$$
\int_{C_{U}}^{C_{L}} \pi(\sigma \mid \mathbf{x}) d \sigma=1-\alpha
$$

and

$$
\pi\left(C_{U} \mid \mathbf{x}\right)=\pi\left(C_{L} \mid \mathbf{x}\right) .
$$

After some algebra, the equations in (17) and (18) take the form

$$
\Gamma\left(\frac{1}{2 C_{L}^{2}}, m+\delta, W\right)-\Gamma\left(\frac{1}{2 C_{U}^{2}}, m+\delta, W\right)=1-\alpha
$$

and

$$
\left(\frac{C_{U}}{C_{L}}\right)^{2(m+\delta)+1}=\exp \left\{-\frac{W}{2}\left(\frac{1}{C_{U}^{2}}-\frac{1}{C_{L}^{2}}\right)\right\}
$$

where $\mathrm{W}=\sum_{i=1}^{m} x_{i: n}^{2}+(n-m) C^{2}+\beta$ and $\Gamma(t, a, b)=\int_{0}^{t} \frac{b^{a}}{\Gamma(a)} y^{a-1} e^{-b y} d y$ is the incomplete gamma function.

\section{Reliability function}

\subsection{Maximum likelihood estimation}

We can easily obtain the MLE of the reliability function $R(t \mid \sigma)$ by the invariance property of the MLE. That is,

$$
\hat{R}_{M}=R\left(t \mid \hat{\sigma}_{M}\right)=\exp \left(-\frac{t^{2}}{2 \hat{\sigma}_{M}^{2}}\right)
$$

\subsection{Bayesian estimation}

Let $R=R(t \mid \sigma)$. Then, we can obtain the posterior distribution $\pi(R \mid \mathbf{x})$ by substituting $\sigma=\sqrt{\frac{t^{2}}{-2 \ln R}}$ into the posterior distribution (12).

$\pi(R \mid \mathbf{x}) \propto \frac{1}{\Gamma(m+\delta)}\left(\frac{\sum_{i=1}^{m} x_{i: n}^{2}+(n-m) C^{2}+\beta}{t^{2}}\right)^{m+\delta}(-\ln R)^{m+\delta-1} R^{\frac{\sum_{i=1}^{m} x_{i: n}^{2}+(n-m) C^{2}+\beta}{t^{2}}-1}$.

From the posterior distribution (22), the Bayes estimator of $R(t \mid \sigma)$ under the squared loss function is given by

$$
\hat{R}_{B}=E(R \mid \mathbf{x})=\left(\frac{\sum_{i=1}^{m} x_{i: n}^{2}+(n-m) C^{2}+\beta}{t^{2}+\sum_{i=1}^{m} x_{i: n}^{2}+(n-m) C^{2}+\beta}\right)^{m+\delta} .
$$

On differentiating the log-posterior distribution with respect to $R$ in turn and setting the equation to zero, we obtain the equation as

$$
\begin{aligned}
\frac{\partial \ln \pi(R \mid \mathbf{x})}{\partial R} & =\frac{\delta+m-1}{R \ln R}+\frac{\sum_{i=1}^{m} x_{i: n}^{2}+(n-m) C^{2}+\beta-t^{2}}{R t^{2}} \\
& =0 .
\end{aligned}
$$


Then, we can find the HPD estimator by solving equation (24).

$$
\hat{R}_{H}=\exp \left(-\frac{t^{2}(m+\delta-1)}{\sum_{i=1}^{m} x_{i: n}^{2}+(n-m) C^{2}+\beta-t^{2}}\right)
$$

\section{Entropy}

\subsection{Maximum likelihood estimation}

We can easily obtain the MLE of entropy $H(f)$ by the invariance property of the MLE. That is,

$$
\hat{H}_{M}=1+\ln \left(\frac{\hat{\sigma}_{M}}{\sqrt{2}}\right)+\frac{\gamma}{2}
$$

\subsection{Bayesian estimation}

Let $H=H(f)$. Then, we can obtain the posterior distribution $\pi(H \mid \mathbf{x})$ by substituting $\sigma=\sqrt{2} e^{\left(H-1-\frac{\gamma}{2}\right)}$ into the posterior distribution (12).

$\pi(H \mid \mathbf{x}) \propto \frac{2}{\Gamma(m+\delta)}\left(\frac{\sum_{i=1}^{m} x_{i: n}^{2}+(n-m) C^{2}+\beta}{\left(2 e^{H-1-\frac{\gamma}{2}}\right)^{2}}\right)^{m+\delta} \exp \left(-\frac{\sum_{i=1}^{m} x_{i: n}^{2}+(n-m) C^{2}+\beta}{\left(2 e^{H-1-\frac{\gamma}{2}}\right)^{2}}\right)$.

Since the posterior distribution (27) is complicated for the entropy $H$, we can't easily obtain the Bayes estimator. Instead, we can obtain the Bayes estimator using the method proposed by Tiernery and Kandane (1986).

$$
E[g(H) \mid \mathbf{x}]=\int g(H) \pi(H \mid \mathbf{x}) d H=\frac{\int e^{n l^{*}(H)} d H}{\int e^{n l(H)} d H}
$$

where

$$
l(H)=\frac{1}{n} \ln \pi(H \mid \mathbf{x}), \quad l^{*}(H)=\left(l(H)+\frac{1}{n} \ln g(H)\right) .
$$

Tiernery and Kandane (1986) showed that the expectation (28) can be approximated as

$$
E[g(H) \mid \mathbf{x}] \approx\left(\frac{\left|\psi^{*}\right|}{|\psi|}\right)^{\frac{1}{2}} \exp \left(n\left\{l^{*}\left(\hat{H}^{*}\right)-l(\hat{H})\right\}\right)=\left(\frac{\left|\psi^{*}\right|}{|\psi|}\right)^{\frac{1}{2}} \frac{g\left(\hat{H}^{*}\right) \pi\left(\hat{H}^{*} \mid \mathbf{x}\right)}{\pi(\hat{H} \mid \mathbf{x})}
$$

where $\hat{H}^{*}$ and $\hat{H}$ maximize $l^{*}(H)$ and $l(H)$, respectively and $\psi^{*}$ and $\psi$ are minus the inverse of the second derivatives of $l^{*}(H)$ and $l(H)$ at $\hat{H}^{*}$ and $\hat{H}$, respectively. Since the Bayes estimator 
is the mean of the posterior distribution under the squared error loss function, $g(H)=H$ and

$$
\begin{aligned}
l(H)= & \frac{1}{n}\left[\ln K+(m+\delta) \ln \left(\sum_{i=1}^{m} x_{i: n}^{2}+(n-m) C^{2}+\beta\right)-(m+\delta)\left\{\ln 4+2\left(H-1-\frac{\gamma}{2}\right)\right\}\right. \\
& \left.-\frac{\sum_{i=1}^{m} x_{i: n}^{2}+(n-m) C^{2}+\beta}{4 e^{2\left(H-1-\frac{\gamma}{2}\right)}}\right], \\
l\left(H^{*}\right)= & \left(l(H)+\frac{1}{n} \ln H\right)
\end{aligned}
$$

where $K$ is a constant. We can obtain the Bayes estimator by substituting equation (30) into equation (29) as follows.

$$
\hat{H}_{B}=\left(\frac{\left|\psi^{*}\right|}{|\psi|}\right)^{\frac{1}{2}} \frac{\hat{H}^{*} \exp \left\{-2\left(\hat{H}^{*}-1-\frac{\gamma}{2}\right)(m+\delta)\right\} \exp \left(-\frac{\sum_{i=1}^{m} x_{i: n}^{2}+(n-m) C^{2}+\beta}{4 e^{2\left(\hat{H}^{*}-1-\frac{\gamma}{2}\right)}}\right)}{\exp \left\{-2\left(\hat{H}-1-\frac{\gamma}{2}\right)(m+\delta)\right\} \exp \left(-\frac{\sum_{i=1}^{m} x_{i: n}^{2}+(n-m) C^{2}+\beta}{4 e^{2\left(\hat{H}-1-\frac{\gamma}{2}\right)}}\right)} .
$$

To obtain the HPD estimator, we can use equation (27). On differentiating the log-posterior distribution (27) with respect to $H$ in turn and setting the equation to zero, we obtain the equation as

$$
\frac{\partial \ln \pi(H \mid \mathbf{x})}{\partial H}=-2(m+\delta)+\frac{\sum_{i=1}^{m} x_{i: n}^{2}+(n-m) C^{2}+\beta}{2} \exp \left(-2\left(H-1-\frac{\gamma}{2}\right)\right)=0 .
$$

We can obtain the HPD estimator $\hat{H}_{H}$ by solving equation (32).

$$
\hat{H}_{H}=1+\frac{\gamma}{2}-\frac{1}{2} \ln \left(\frac{4(m+\delta)}{\sum_{i=1}^{m} x_{i: n}^{2}+(n-m) C^{2}+\beta}\right)
$$

\section{Illustration example and simulation results}

\subsection{Illustration example}

In this section, we use the following real data presented in Lieblein and Zelen (1956). They performed life tests and determined the number of revolutions to failure for 23 ball bearings. The observed failure times are as follows:

Table 1: Real data set

\begin{tabular}{llllllllll}
\hline 0.1788 & 0.2892 & 0.3300 & 0.4152 & 0.4212 & 0.4560 & 0.4848 & 0.5184 & 0.5196 & 0.5412 \\
0.5556 & 0.6780 & 0.6864 & 0.6864 & 0.6888 & 0.8412 & 0.9312 & 0.9864 & 1.0512 & 1.0584 \\
1.2792 & 1.2804 & 1.7340 & & & & & & & \\
\hline
\end{tabular}

For this data set, Raqab and Madi (2002) indicated that the one-parameter Rayleigh distribution provides a satisfactory fit. In this example, we assume that the underlying distribution of this data is the Rayleigh distribution based on the unified hybrid censoring scheme. We take Case I $\left(r=8, k=5, T_{1}=0.6, T_{2}=1.0\right)$, Case II $\left(r=16, k=7, T_{1}=0.6, T_{2}=1.0\right)$, Case III $\left(r=20, k=6, T_{1}=0.6, T_{2}=1.0\right)$, Case IV $\left(r=16, k=12, T_{1}=0.6, T_{2}=1.0\right)$, Case V $(r=20, k=12$, 
Table 2: Estimation of the scale parameter for the example

\begin{tabular}{cccccc|ccc|ccc|ccc}
\hline Case & $n$ & $r$ & $k$ & $T_{1}$ & $T_{2}$ & $\hat{\sigma}_{M}$ & $\hat{\sigma}_{B}$ & $\hat{\sigma}_{H}$ & $\hat{R}_{M}$ & $\hat{R}_{B}$ & $\hat{R}_{H}$ & $\hat{H}_{M}$ & $\hat{H}_{B}$ & $\hat{H}_{H}$ \\
\hline 1 & 8 & 5 & & & 0.5426 & 0.6599 & 0.6319 & 0.6541 & 0.7412 & 0.7499 & 0.3307 & 0.5177 & 0.4999 \\
2 & 16 & 7 & & & 0.5504 & 0.6365 & 0.6163 & 0.6619 & 0.7274 & 0.7338 & 0.3450 & 0.4840 & 0.4707 \\
3 & 20 & 6 & 0.6 & 1.0 & 0.5672 & 0.6415 & 0.6231 & 0.6780 & 0.7317 & 0.7375 & 0.3750 & 0.4924 & 0.4804 \\
4 & 23 & 16 & 12 & & & 0.5504 & 0.6365 & 0.6163 & 0.6619 & 0.7274 & 0.7338 & 0.3450 & 0.4840 & 0.4707 \\
5 & 20 & 12 & & & 0.5672 & 0.6415 & 0.6231 & 0.6780 & 0.7317 & 0.7375 & 0.3750 & 0.4924 & 0.4804 \\
6 & 21 & 19 & & & 0.5644 & 0.6362 & 0.6187 & 0.6755 & 0.7282 & 0.7337 & 0.3701 & 0.4843 & 0.4728 \\
\hline
\end{tabular}

$\left.T_{1}=0.6, T_{2}=1.0\right)$, and Case VI $\left(r=21, k=19, T_{1}=0.6, T_{2}=1.0\right)$. For the Bayesian inference, the prior parameters are chosen $(\delta, \beta)=(3.5,5.5)$. Table 1 reports results for the real data.

\subsection{Simulation results}

To compare the performance of all previously proposed estimators for the scale parameter, reliability function, and entropy, we simulate the biases and the MSEs of all proposed estimators by using the Monte Carlo simulation. The average lengths of the CrIs is obtained by 10,000 sampling from the posterior distribution. The unified hybrid censored samples from the Rayleigh distribution are generated for sample size $n=20$ and 40 . Using these samples, the MSEs of all proposed estimators are obtained with $\sigma=1$. The simulation run 10,000 times. For the reliability function, we set $t=0.5$. For the Bayesian inference, the prior parameters are chosen $(\delta, \beta)=(3.5,5.5)$.

We also derive the asymptotic relative efficiency (ARE) between the two estimators. ARE is helpful for comparing the performance of the Bayes estimator and HPD estimator with the MLE. The ARE is defined as follows:

$$
\operatorname{ARE}\left(\hat{\sigma}^{*}, \hat{\sigma}\right)=\frac{\operatorname{MSE}(\hat{\sigma})}{\operatorname{MSE}\left(\hat{\sigma}^{*}\right)},
$$

where the $\operatorname{MSE}(\hat{\sigma})$ denotes the MSE, which is estimated by $\sum_{i=1}^{n}\left(\hat{\sigma}_{i}-\sigma\right)^{2} / n$. If $\operatorname{ARE}\left(\hat{\sigma}^{*}, \hat{\sigma}\right)>1$, $\hat{\sigma}^{*}$ is more efficient than $\hat{\sigma}$. All results are given in Tables $2 \sim 5$.

The results in the Tables reveal that for all proposed estimators, the MSEs generally decrease as the sample size $n$ increases. When the sample size $n$ is fixed, the MSEs generally decrease as the object number that must be observed $k$ increases. When the sample size $n$ and the object number that must be observed $k$ are both fixed, the MSEs generally decrease as the predetermined observation number $r$ increases. Lastly, the MSEs generally decrease as the predetermined end time $T_{1}$ and the extended end time $T_{2}$ increase.

These simulation results demonstrate that the HPD estimator and the Bayes estimator are more efficient than the MLE in the sense of the MSE. Likewise, the HPD estimator and the Bayes estimator are more efficient than the MLE for ARE. For reliability function, the HPD estimator is a more efficient estimator than the other two estimators in the ARE. We also show that the Bayes estimator obtained by using the mean of the posterior distribution is more efficient than the other two estimators for entropy in the ARE. The average length of CI based on the MLE is longer than the lengths of intervals based on the others.

\section{Conclusion}

We derive some estimators of the scale parameter of the Rayleigh distribution based on unified hybrid censoring. We also estimate the reliability function and the entropy for the Rayleigh distribution. First, we obtain the MLE. Second, we obtain the Bayes estimator by using the natural conjugate prior distribution. Lastly, we obtain the HPD estimator based on the maximum likelihood principle by using the natural conjugate prior distribution. 
Table 3: The MSEs(biases) and asymptotic relative efficiencies (ARE) of the proposed estimators of $\sigma$

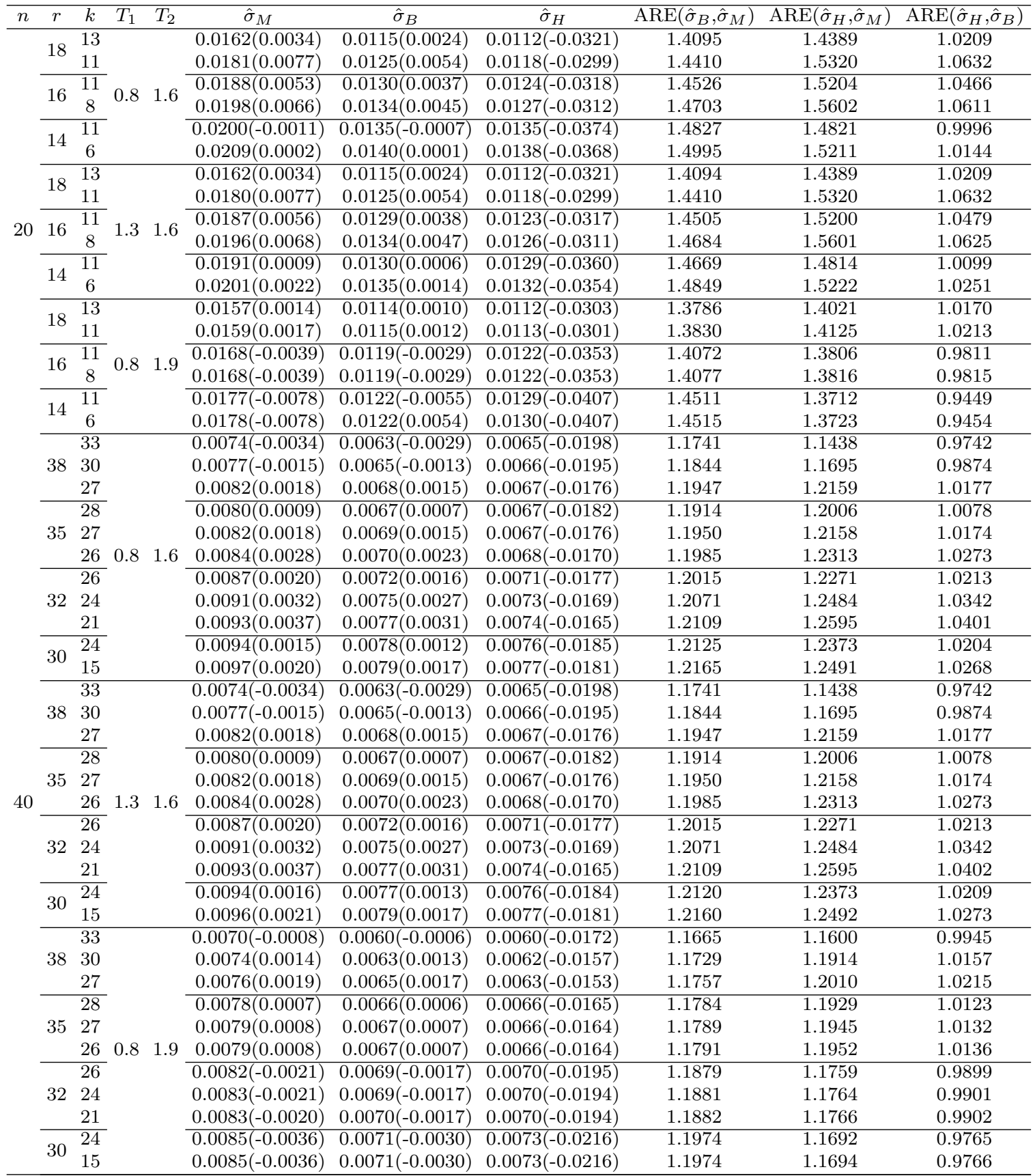


Table 4: The MSEs(biases) of the estimators of the reliability function

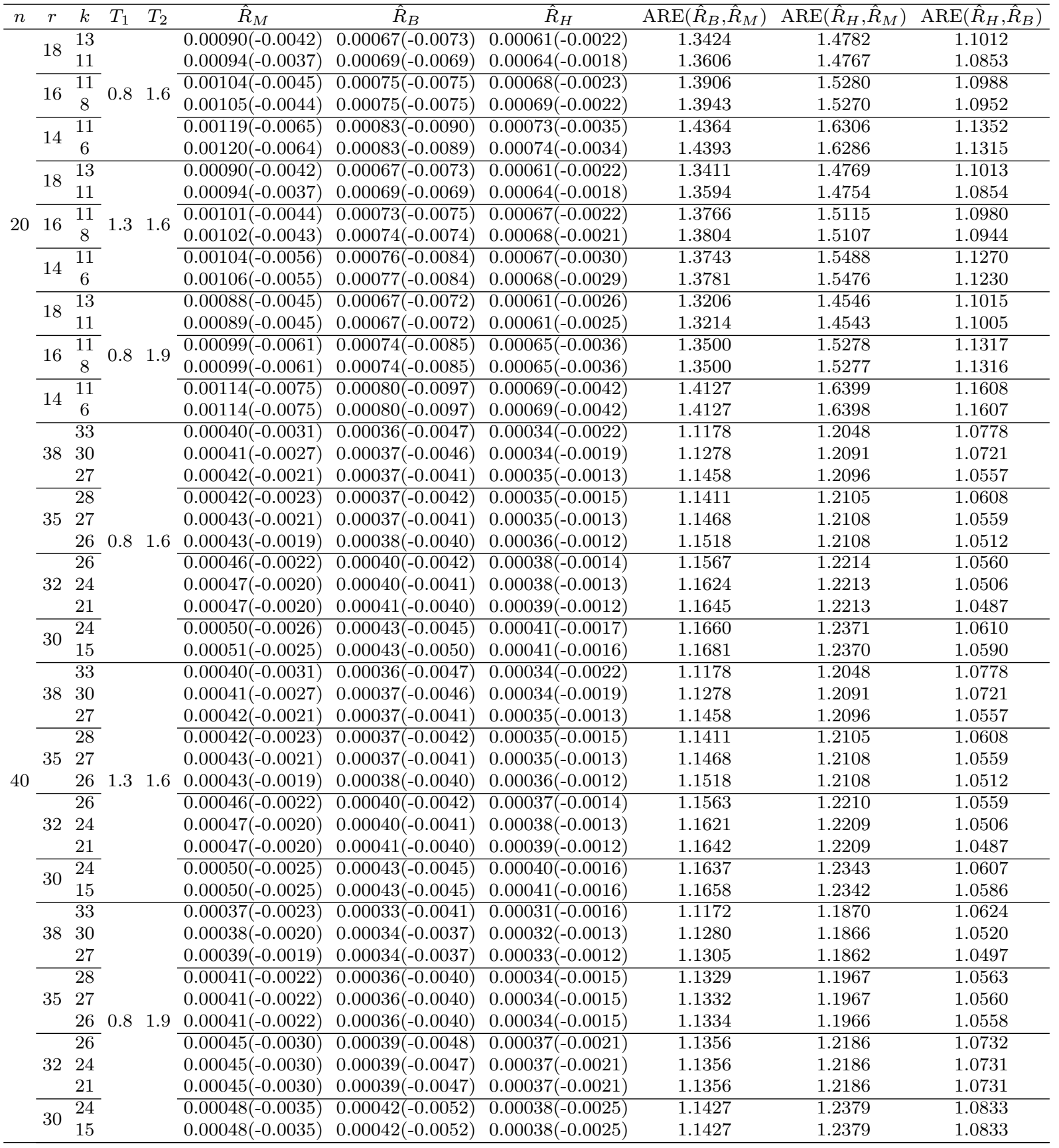


Table 5: The MSEs(biases) of the estimators of the entropy

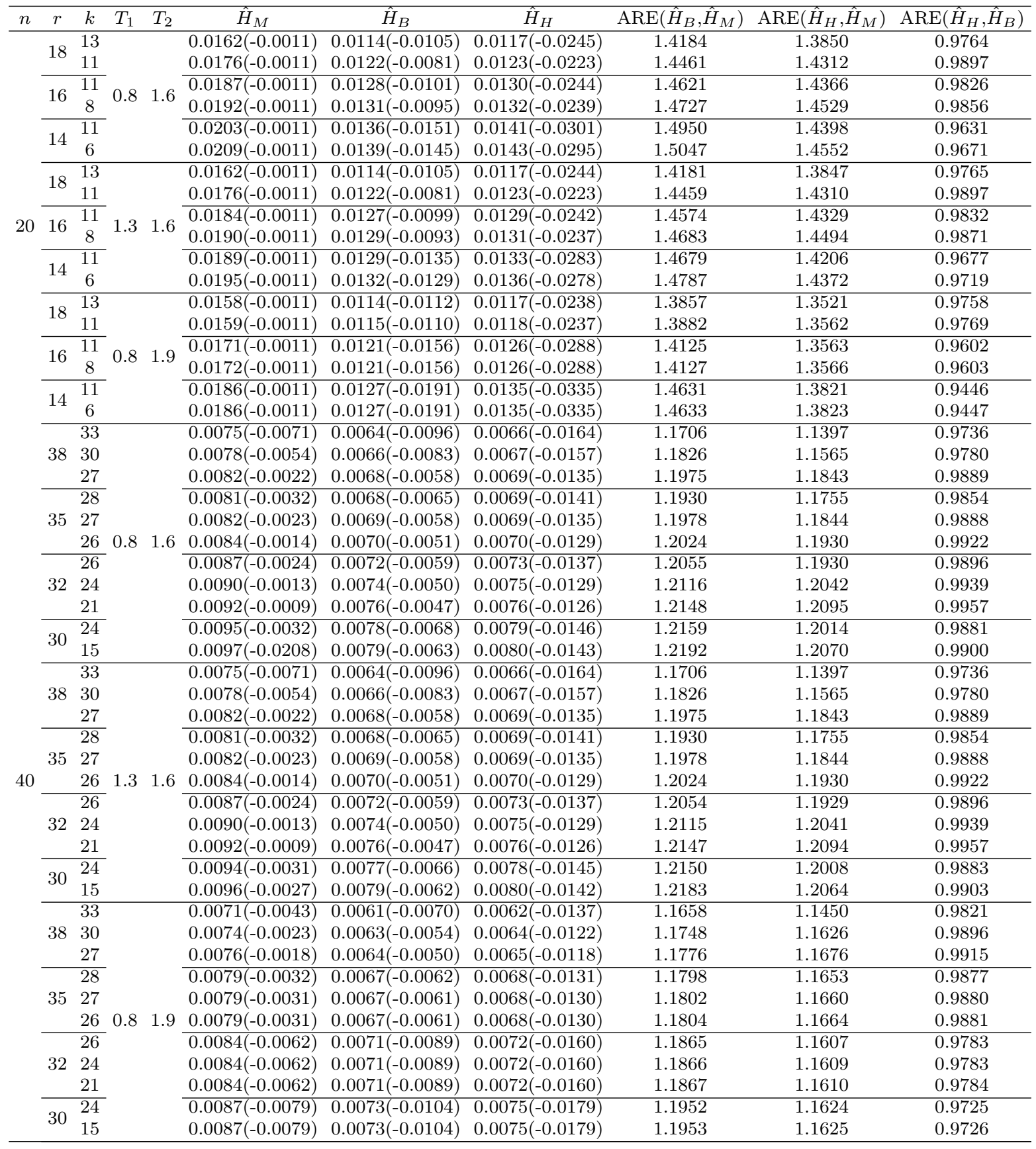


Table 6: Coverage probabilities(average lengths of 95\% \& 90\% interval)

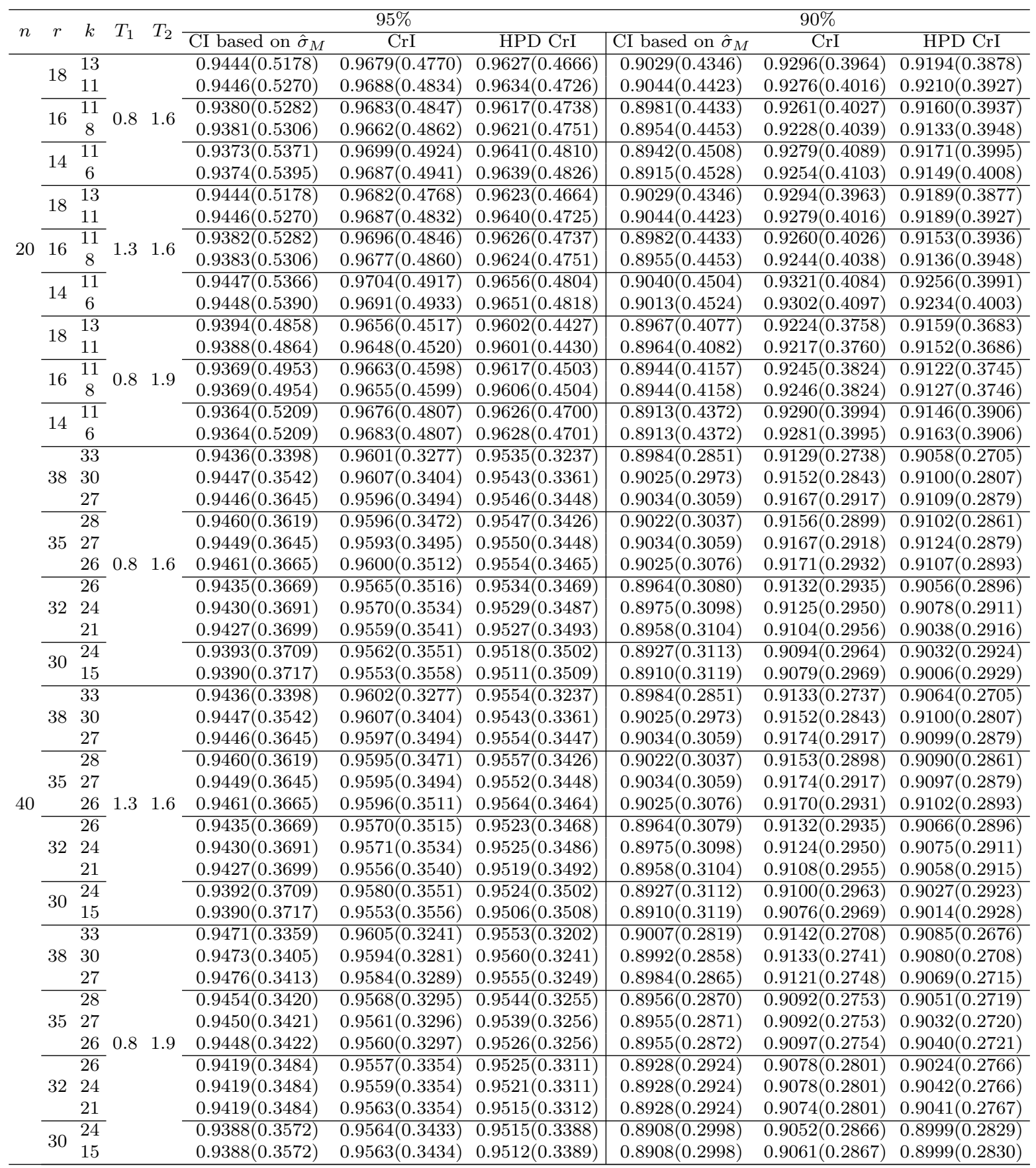


The Bayes estimators has better performance than MLE. Especially, the estimator $\hat{R}_{H}$ is more efficient than the estimator $\hat{R}_{B}$ for reliability function in the ARE and the estimator $\hat{H}_{B}$ is more efficient than the estimator $\hat{H}_{H}$ for entropy in the ARE.

In this paper, we developed the estimation method for the reliability function and entropy based on the complete sample because the estimators of the reliability function and the entropy are not easily obtained under unified hybrid censoring scheme. To obtain the estimators of reliability function and entropy under unified hybrid censoring scheme, we need more study.

\section{References}

Abdel-Hamid AH, AL-Hussaini EK (2014). "Bayesian Prediction for Type-II Progressivecensored Data from the Rayleigh Distribution under Progressive-stress Model." Journal of Statistical Computation and Simulation, 84, 1297-1312.

Ateya SF (2017). "Estimation under Inverse Weibull Distribution Based on Balakrishnans Unified Hybrid Censored Scheme." Communications in Statistics-Simulation and Computation, 46, 3645-3666.

Balakrishnan N (1989). "Approximate MLE of the Scale Parameter of the Rayleigh Distribution with Censoring." IEEE Transactions on Reliability, 38, 355-357.

Balakrishnan N, Rasouli A, Sanjari Farsipour N (2008). "Exact Likelihood Inference Based on an Unified Hybrid Censored Sample from the Exponential Distribution." Journal of Statistical Computation and Simulation, 78, 475-488.

Chandrasekar B, Childs A, Balakrishnan N (2004). "Exact Likelihood Inference for the Exponential Distribution under Generalized Type-I and Type-II Hybrid Censoring." Naval Research Logistics (NRL), 51, 994-1004.

Chaturvedi A, Kang SB, Pathak A (2016). "Estimation and Testing Procedures for the Reliability Functions of Generalized Half Logistic Distribution." Journal of The Korean Statistical Society, 45, 314-328.

Childs A, Chandrasekar B, Balakrishnan N, Kundu D (2003). "Exact Likelihood Inference Based on Type-I and Type-II Hybrid Censored Samples from the Exponential Distribution." Annals of the Institute of Statistical Mathematics, 55, 319-330.

Cho Y, Sun H, Lee K (2014). "An Estimation of the Entropy for a Rayleigh Distribution Based on Doubly-generalized Type-II Hybrid Censored Samples." Entropy, 16, 3655-3669.

Dey T, Dey S, Kundu D (2016). "On Progressively Type-II Censored Two-parameter Rayleigh Distribution." Communications in Statistics-Simulation and Computation, 45, 438-455.

Fernandez AJ (2000). "Bayesian Inference from Type II Doubly Censored Rayleigh Data." Statistics $\&$ Probability Letters, 48, 393-399.

Han JT, Kang SB (2006). "Estimation for Two-parameter Rayleigh Distribution Based on Multiply Type-II Censored Sample." Journal of the Korean Data and Information Science Society, 17, 1319-1328.

Kang SB, Cho YS, Han JT, Kim J (2012). "An Estimation of the Entropy for a Double Exponential Distribution Based on Multiply Type-II Censored Samples." Entropy, 14, 161173.

Kaushik A (2019). "A Progressive Interval Type-I Censored Life Test Plan for Rayleigh Distribution." Austrian Journal of Statistics, 48, 76-86. 
Kim C, Han K (2009). "Estimation of the Scale Parameter of the Rayleigh Distribution under General Progressive Censoring." Journal of the Korean Statistical Society, 38, 239-246.

Kundu D, Raqab MZ (2015). "Estimation of $R=P[Y<X]$ for Three-parameter Generalized Rayleigh Distribution." Journal of Statistical Computation and Simulation, 85, 725-739.

Lieblein J, Zelen M (1956). "Statistical Investigation of the Fatigue Life of Deep-groove Ball Bearings." Journal of Research of the National Bureau of Standards, 57, 273-316.

Panahi H, Sayyareh A (2016). "Estimation and Prediction for a Unified Hybrid-censored Burr Type XII Distribution." Journal of Statistical Computation and Simulation, 86, 55-73.

Raqab M, Madi M (2002). "Bayesian Prediction of the Total Time on Test Using Doubly Censored Rayleigh Data." Journal of Statistical Computation and Simulation, 72, 781-789.

Raqab MZ, Madi MT (2011). "Inference for the Generalized Rayleigh Distribution Based on Progressively Censored Data." Journal of Statistical Planning and Inference, 141, 33133322.

Seo JI, Jeon JW, Kang SB (2016). "Exact Interval Inference for the Two Parameter Rayleigh Distribution Based on the Upper Record Values." Jouranl of Probability and Statistics, 2016.

Seo JI, Kang SB (2015). "Bayesian Estimation of the Entropy of the Half-Logistic Distribution Based on Type-II Censored Samples." International Journal of Applied Mathematics and Statistics, 53, 58-66.

Seo JI, Kang SB (2017). "More Efficient Inference Using Hybrid Censored Data from a Continuous Probability Distribution with the Scale Parameter." Far East Journal of Mathematical Sciences, 101, 2043-2054.

Tiernery L, Kandane JB (1986). "Accurate Approximations for Posterior Moments and Marginal Densities." Journal of American Statistical Association, 81, 82-86.

Wu S, Chen D, Chen S (2006). "Bayesian Inference for Rayleigh Distribution under Progressive Censored Sample." Applied Stochastic Models in Business and Industry, 22, 269-279.

\author{
Affiliation: \\ Young Eun Jeon \\ Department of Statistics, \\ Yeungnam University \\ Gyeongsan 38541, Republic of Korea \\ Suk-Bok Kang \\ Professor \\ Department of Statistics, \\ Yeungnam University \\ Gyeongsan 38541, Republic of Korea \\ Corresponding Author \\ E-mail: sbkang@yu.ac.kr
}

\section{Austrian Journal of Statistics}

published by the Austrian Society of Statistics

Volume 50

January 2021 http://www.ajs.or.at/

http://www.osg.or.at/

Submitted: 2019-06-26

Accepted: 2019-10-28 